\title{
Conséquences sur le progrès génétique laitier d'une sélection sur des caractères secondaires chez les bovins
}

\author{
V. DUCROCQ \\ I.N.R.A., Station de Génétique quantitative et appliquée, \\ Centre de Recherches zootechniques, F 78350 Jouy-en-Josas
}

\begin{abstract}
Résumé
L'algorithme d'intégration numérique d'une loi multinormale proposé par Durr $(1973,1975)$ rend possible le calcul du progrès génétique après sélection successive sur plusieurs caractères à partir de la relation exacte de JAIN \& AMBLE (1962), sans les classiques limitations concernant le nombre d'étapes et de critères de sélection retenus et quelles que soient les corrélations génétiques entre ces caractères. Son utilisation dans le cadre d'un schéma théorique de sélection chez les bovins laitiers a permis l'étude de l'impact sur la production laitière d'une sélection sur un ou plusieurs caractères secondaires, comme la vitesse de croissance, la conformation bouchère ou la conformation laitière (type). Il apparaît que la perte de progrès génétique laitier annuel entraînée par cette sélection, et mesurée par rapport à un schéma maximaliste ne s'intéressant qu'au caractère principal, varie couramment entre 1,5 et 15 p. 100 suivant le contexte, les corrélations génétiques entre caractères et les pressions de sélection appliquées. Cette perte croît sensiblement : a) lorsqu'augmente la pression de sélection sur le caractère secondaire (et ceci de façon non linéaire) ; b) lorsque le schéma considéré est globalement peu intensif en ce qui concerne la sélection sur la production laitière ; $\mathrm{c}$ ) lorsque la répartition des efforts de sélection selon les différentes voies de transmission du patrimoine génétique d'une génération à la suivante est déséquilibrée ; d) lorsque le caractère secondaire est défavorablement lié à la production laitière, même très faiblement $\left(r_{G}=-0,10\right)$. Ce dernier point particulièrement net, et plus généralement l'ensemble de ces résultats, soulignent la nécessité d'une limitation très stricte des objectifs de sélection aux seuls caractères ayant un intérêt économique certain.
\end{abstract} daires.

Mots clés : Progrès génétique, corrélation génétique, production laitière, bovins, caractères secon-

\section{Summary}

\section{Effects of selection for secondary traits on genetic gain for milk production in cattle}

After successive selection on several traits using the exact relationship of JAIN \& AMBLE (1962), genetic gain could be computed, by the algorithm of numerical integration of a multinormal distribution proposed by DUTT $(1973,1975)$. It reduces the usual restrictions on the number of stages and of selection criteria used. Using this algorithm in a theoretical selection scheme for dairy cattle, it was possible to study the impact on milk production of the selection for one or more secondary traits such as growth rate, beef conformation and dairy type. The loss in annual genetic gain for milk yield due to this selection and measured in comparison with a plan maximizing only the main trait, generally varied between 1.5 and $15 \mathrm{p}$. 100 , depending on the context, genetic relationships between traits and applied selection intensity. This loss increased markedly when : a) the selection intensity on the secondary trait increased (in a non-linear way) ; b) the selection 
on milk production in the plan considered was less intensive ; c) the distribution of selection efforts according to the different pathways of genetic inheritance from one generation to the next was unbalanced; d) the secondary trait was unfavorably linked to milk production, even for low correlations. This last point, which was particularly clear, and all these results in general, emphasize the need to limit selection objectives to only those characters which are definitely of economic value.

Key words: Genetic gain, genetic correlation, milk yield, cattle, secondary traits.

\section{Introduction}

L'un des aspects les plus nets de l'évolution actuelle des exploitations laitières en France, et plus généralement en Europe de l'Ouest, réside dans les modifications génétiques du cheptel entraînées par le développement rapide et irréversible (COLLEAU, 1982c), par croisement, d'animaux de type spécialisé (Holstein Friesian, Brown Swiss) à l'intérieur de races d'aptitudes plus mixtes. Par ailleurs, les objectifs de sélection semblent s'être concentrés sur les caractères de production laitière incontestablement plus avantageux dans le contexte économique actuel, aux dépens de l'intérêt accordé à certains caractères dits " secondaires » (à l'exception toutefois des caractères regroupés sous le terme général de "type " ou conformation laitière).

Or les caractères secondaires présentant un intérêt économique certain sont nombreux (MAijala, 1974 ; Philipsson et al., 1975 ; Vinson, 1982). Outre tous les aspects liés à la production de viande (croissance, efficacité alimentaire, conformation bouchère), on peut citer certains points de l'appréciation de la conformation laitière (mamelle, aplombs), la vitesse de traite, les performances de reproduction, la résistance à certaines maladies, etc.

Au cours des 20 dernières années, divers auteurs ont tenté d'intégrer la sélection de caractères autres que la production laitière dans l'étude de la rentabilité globale des schémas de sélection chez les bovins laitiers dans le but de mettre en évidence des procédures optimales à cet égard (SOLLER et al., 1966 ; LiNDHE, 1968 ; HINKS, 1970 ; Hill, 1971 ; Brascamp, 1974 ; Niebel, 1974 ; Petersen et al., 1974 ; Cunningham \& Mc Clintock, 1974 ; Depotter \& Vandepitte, 1980 ; Cunningham \& Moioli, 1982). La plupart de ces travaux a porté sur la sélection d'un seul caractère secondaire (la vitesse de croissance), en une seule étape (après contrôle individuel des jeunes taurillons en station), ce caractère étant supposé génétiquement indépendant de la production laitière. BRASCAMP (1974) et CunNinghaM \& MC ClinToCK (1974) ont envisagé un plus large éventail de corrélations génétiques entre caractères principal et secondaire (de $-0,2$ à $+0,2$ ). Enfin, seuls Soller et al. (1966), NiEbEl (1974), CuNNINGHAM \& MoIOLI (1982) ont étudié l'intérêt d'une sélection du caractère secondaire en 2 étapes (contrôle individuel puis contrôle sur descendance).

Le présent article vise à étudier l'impact global de la sélection des caractères secondaires dans les schémas d'amélioration génétique des bovins laitiers typiques de la France. Par rapport à l'ensemble des travaux précédemment cités, la perspective adoptée est à la fois plus simple au niveau économique et plus détaillée au niveau de la description des conséquences génétiques de ces schémas de sélection.

L'utilisation de l'argumentation économique vis-à-vis de la sélection sur des caractères secondaires peut amener à des résultats contradictoires, qui ne sont pas toujours clairement explicables par des différences de méthodologie : ainsi, CunNINGHAM \& 
MoIOLI (1982) trouvent que la sélection après contrôle individuel est la phase la plus efficace du schéma. A l'inverse, BrascAmp (1974) trouve que le contrôle individuel peut être totalement inefficace, notamment s'il y a opposition génétique entre vitesse de croissance et production laitière $\left(\mathrm{r}_{\mathrm{G}}=-0,2\right)$. En outre, une optimisation économique stricte des schémas peut très bien conduire à la dégradation continue de certains caractères secondaires. Il suffit pour cela que leur importance relative dans l'objectif de sélection soit faible et que le coefficient de corrélation génétique avec la production laitière soit nettement négatif. Or cette dégradation peut être préjudiciable à l'intérêt national à long terme, l'objectif de sélection privilégiant en général l'intérêt à court terme de l'éleveur.

L'approche économique classique peut, par ailleurs, être rejetée par les sélectionneurs, surtout dans le contexte actuel d'orientation laitière très marquée : leur base de référence a tendance à devenir - de facto - le progrès génétique laitier. Ainsi les sélectionneurs considéreront en priorité, non pas le gain économique apporté par la sélection sur un caractère secondaire, mais la perte de progrès génétique laitier occasionnée par cette sélection, perte qu'il faudra donc minimiser.

En conséquence, notre objectif a été d'approfondir la mesure de cette diminution, en l'adaptant mieux à la réalité complexe des schémas de sélection, c'est-à-dire que nous avons étudié des schémas pouvant éventuellement comporter plus de 2 étapes de sélection concernant plus de 2 caractères économiquement intéressants, et souvent interdépendants. L'impact de la sélection simultanée de plusieurs caractères n'a pas été mesuré de façon globale (approche classique à partir de pondérations économiques approximatives) mais caractère par caractère, en référence à un schéma maximaliste (pour lequel la totalité de l'effort de sélection est portée sur la production laitière), bien perçu par les sélectionneurs. De cette manière, il est possible de raisonner empiriquement la sélection des caractères secondaires par une recherche des schémas limitant au maximum la chute du progrès génétique laitier pour un objectif donné.

Pour cette étude, nous avons appliqué la relation exacte (sous les hypothèses classiques de normalité), proposée par JAIN \& AMBLE (1962) permettant d'évaluer les conséquences sur un objectif donné d'une sélection en $n$ étapes et utilisée par MocQuOT \& Foulley (1973) dans le cas d'un schéma à 2 étapes sur des bovins à viande. L'extension à plus de 2 étapes a été effectuée en utilisant un algorithme d'intégration numérique proposé par DUTT $(1973,1975)$. On a pu ainsi étudier, par exemple, des schémas avec pour les mâles, sélection successive sur la vitesse de croissance (mesurée en station), la production laitière, le pointage laitier et la vitesse de traite (après contrôle sur descendance), tous ces caractères étant considérés interdépendants.

\section{Matériel et méthodes}

\section{A. Caractères secondaires envisagés}

Bien que la méthode et les programmes de calcul développés permettent d'envisager des schémas comportant un plus grand nombre de caractères sélectionnés et afin d'aboutir de façon claire à des conclusions simples, nous n'avons retenu ici que 2 groupes de caractères secondaires définis de manière volontairement très générale comme étant :

1) un groupe de caractères à héritabilité assez forte $\left(h^{2}=0,40\right)$ éventuellement corrélés négativement à la production laitière $\left(\mathrm{r}_{\mathrm{G}}=0,-0,15,-0,30\right)$ s'exprimant à 
la fois chez les mâles et les femelles et pouvant faire l'objet d'un contrôle individuel en station. Il sera appelé par la suite " critère (s) de croissance et/ou de production de viande » car la majorité de ces critères répond à cette définition (DUTERTRE, 1975 ; MiLLER et al., 1980) ;

2) un groupe de caractères à héritabilité moyenne $\left(h^{2}=0,25\right)$ ne s'exprimant que chez les femelles, faisant l'objet soit d'une sélection massale (vaches), soit d'une sélection sur descendance (taureaux). Certains postes du pointage laitier (note finale, appréciation de la mamelle) (LE MEZEC, 1980 ; COLleAu et al., 1982a) et certaines caractéristiques de traite (temps machine) correspondent grossièrement à cette définition.

\section{B. Base de sélection}

La modélisation mathématique de la sélection et des modalités de transmission des gènes d'une génération à la suivante (le long des 4 voies père-fils, père-fille, mère-fils, mère-fille) a été réalisée pour une unité de sélection théorique d'importance moyenne. Les paramètres génétiques et démographiques de la population considérée, supposée fermée, sont présentés au tableau 1.

\section{TABLEAU 1}

Schéma de référence: paramètres génétiques et démographiques.

Reference scheme: genetic and demographic parameters.

\section{Paramètres génétiques}

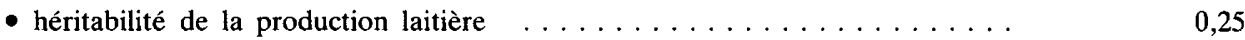

du critère de production de viande $\ldots \ldots \ldots \ldots \ldots \ldots \ldots . \ldots \ldots, 0,40$

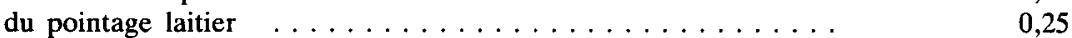

- répétabilité de la production laitière $\ldots \ldots \ldots \ldots \ldots \ldots \ldots \ldots \ldots \ldots$

- corrélation génétique entre la production laitière en $1^{\text {re }}$ lactation et les suivantes 1

- pas d'effet de la consanguinité sur les performances (ou pas d'accroissement du taux de consanguinité)

\section{Paramètres démographiques}

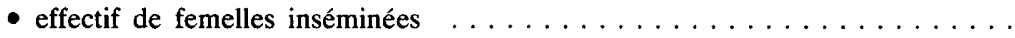

- population active (femelles contrôlées et d'ascendance connue) . . . . . .

- pourcentage de femelles en $1^{\text {re }}$ lactation issues de testage $\ldots \ldots \ldots \ldots$

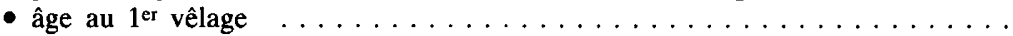

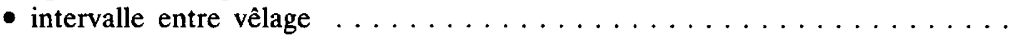

- nombre d'IA nécessaires pour obtenir un veau né vivant $\ldots \ldots \ldots \ldots \ldots$

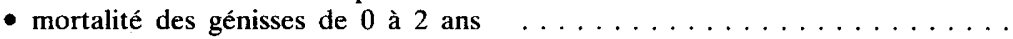

- mortalité des taureaux avant indexation (par an) $\ldots \ldots \ldots \ldots \ldots \ldots$ après indexation (par an) $\ldots \ldots \ldots \ldots \ldots$

- pyramide des âges des femelles (données du contrôle laitier)

\begin{tabular}{|c|c|c|c|c|c|c|c|c|c|c|}
\hline Numéro de lactation & 1 & 2 & 3 & 4 & 5 & 6 & 7 & 8 & 9 & $\overline{10}$ \\
\hline Pourcentage & 26 & 21 & 17 & 13 & 9 & 6 & 4 & 2 & 1 & 1 \\
\hline
\end{tabular}


Un nombre non négligeable des valeurs retenues est à rapprocher de celles utilisées par divers auteurs étudiant la rentabilité de différents schémas de sélection : LINDHE (1968), Hinks (1970a), Brascamp (1974), Salzmann (1978), Depotter \& Vandepitte (1980), Cunningham \& Moioli (1982).

\section{Critères de comparaison}

Deux critères purement génétiques mais pouvant être utilisés pour une éventuelle étude économique ultérieure, ont été choisis pour comparer les différents schémas étudiés. Il s'agit, pour chaque caractère considéré :

- du gain génétique annuel (asymptotique) $\Delta \mathrm{G}_{\mathrm{a}}$

$$
\Delta G_{a}=\frac{\sum_{i} \Delta G_{i}}{\sum_{i} T_{i}} \quad \text { (RENDEL \& RoBERTSON, 1950) }
$$

où $\Delta \mathrm{G}_{\mathrm{i}}$ et $\mathrm{T}_{\mathrm{i}}$ sont la supériorité génétique des parents sur la moyenne des candidats à la sélection, et l'intervalle de génération, pour la voie $i$ (père-fils, père-fille, mère-fils, mère-fille) ;

- du cumul des gains génétiques actualisés, dus à une année de sélection

$$
R=\sum_{t=0}^{s} \theta^{t} \cdot E^{\prime} \cdot X(t) \quad(\text { ELSEN, 1980) }
$$

- $\theta=\frac{1}{1+r} ; \mathbf{r}$ : taux d'actualisation ; s : durée d'actualisation ; l'année $\mathrm{t}$;

- $\mathrm{X}(\mathrm{t})$ est le vecteur des niveaux génétiques des différentes cohortes « âge $\times$ sexe ",

- E est le vecteur $\left(\begin{array}{l}p \\ q\end{array}\right)$ dont les éléments $p_{i}, q_{j}$ sont les « demi »-probabilités pour un animal d'âge 0 l'année $t$ et exprimant le caractère considéré, d'être issu d'un père d'âge $i$ et d'une mère d'âge $j$ l'année $t-1$.

Ce critère $\mathrm{R}$ a l'avantage de tenir compte du délai d'obtention du progrès génétique après les opérations de sélection (par le biais de l'actualisation) et des fluctuations des niveaux génétiques les premières années suivant la mise en place du schéma de sélection (Hill, 1974 ; ElSEN \& MoCQuot, 1974). Comme R ne dépend pratiquement pas de la durée d'actualisation s si celle-ci est supérieure à 20-30 ans, on a pris $s=+\infty$ ce qui simplifie considérablement les calculs (ELSEN, 1980).

L'impact sur le progrès génétique laitier d'une sélection du (ou des) caractère (s) secondaire (s) a été évalué en pourcentage de perte (PP) par comparaison à un schéma laitier maximaliste, tous les schémas supposant un nombre annuel identique de taureaux testés sur descendance (car c'est une contrainte pratique très importante).

$$
P_{G}=\frac{\Delta G_{L M A X}-\Delta G_{L}}{\Delta G_{L M A X}} .100 \text { ou } P_{R}=\frac{\left(R_{L M A X}-R_{L}\right)}{R_{L M A X}} .100
$$

$\Delta G_{\text {LMax }}$ ou $R_{\text {LMax }}$ sont obtenus quand toutes les possibilités de sélection, dans les limites de la contrainte citée plus haut, sont consacrées à l'amélioration de la production laitière. 


\section{Modalités de sélection}

D'un point de vue pratique, on a considéré que la sélection était réalisée par étapes (c'est-à-dire successivement) et à niveaux indépendants (c'est-à-dire séparément) sur chacun des caractères : ne sont retenus que les animaux dont les valeurs génétiques estimées sont supérieures pour tous les critères de sélection à des seuils (points de troncature) dépendant du pourcentage d'animaux conservés à chaque étape.

\section{E. Supériorité des animaux sélectionnés}

Le calcul de la supériorité génétique moyenne $\Delta G_{\mathrm{v}}$ pour le caractère $Y$ des animaux retenus après $n$ étapes de sélection sur les critères $X_{1}, \ldots, X_{n}$ présente 2 difficultés :

1) la détermination des points de troncature $k_{i}$, connaissant la proportion $\alpha_{i}$ d'animaux conservés à chaque étape i c'est-à-dire tels que :

$$
\alpha_{1} \ldots \alpha_{i}=\int_{k_{1}}^{\infty} \ldots \int_{k_{i}}^{\infty} f_{1}\left(x_{1}, \ldots, x_{i}\right) d x_{1} \ldots d x_{i}
$$

où $\mathrm{i} \in[1, \mathrm{n}]$ et $\mathrm{f}_{1}\left(\mathrm{x}_{1}, \ldots, \mathrm{x}_{\mathrm{i}}\right)$ est la densité de la loi conjointe des variables $\mathrm{X}_{1}, \ldots, \mathrm{X}_{\mathrm{i}}$.

2) la détermination de $\Delta G_{y}$ proprement dite :

$$
\Delta G_{y}=\frac{1}{\alpha_{1} \ldots \alpha_{n}} \int_{-\infty}^{\infty} y d y \int_{k_{1}}^{\infty} \ldots \int_{k_{n}}^{\infty} f_{2}\left(y, x_{1}, \ldots, x_{n}\right) d x_{1} \ldots d x_{n}
$$

où $\mathrm{f}_{2}\left(\mathrm{y}, \mathrm{x}_{1}, \ldots, \mathrm{x}_{\mathrm{n}}\right)$ est la densité de la loi conjointe des variables $\mathrm{Y}, \mathrm{X}_{1}, \ldots, \mathrm{X}_{\mathrm{n}}$.

Nous avons fait l'hypothèse que cette loi est multinormale et le reste au cours des générations de sélection. On peut de plus supposer sans perte de généralité qu'elle est centrée et réduite (chaque variable est exprimée en écart-type par rapport à la moyenne). Généralisant les résultats de Cochran (1950), obtenus dans le cas de 2 étapes de sélection, JAIN \& AMBLE (1962) ont montré qu'en cas de loi multinationale :

$$
\Delta G_{y}=\frac{\varrho_{1} Z_{1} I_{2,3, \ldots, n}+\ldots+\varrho_{n} Z_{n} I_{1,2, \ldots, n-1}}{\alpha_{1} \alpha_{2} \ldots \alpha_{n}}
$$

où $\bullet \varrho_{1} \ldots \varrho_{n}$ sont les corrélations simples entre $Y$ et $X_{1}, X_{2}, \ldots, X_{n}$;

- $\mathrm{Z}_{\mathrm{i}}$ est l'ordonnée de la densité de la loi normale en $\mathrm{k}_{\mathrm{i}}$ (seuil de sélection);

- $\mathrm{I}_{2, \ldots, \mathrm{n}}$ est le volume incomplet occupé par les points de la distribution multinormale des $(n-1)$ variables $X_{2}, \ldots, X_{n}$ tels que $x_{2}>h_{2}, \ldots, x_{n}>h_{n}$ avec:

$$
\begin{gathered}
h_{i}=\frac{k_{i}-\varrho_{i 1} k_{1}}{\sqrt{1-\varrho_{i I}^{2}}} ; \varrho_{i 1}=\frac{\operatorname{cov}\left(X_{i}, X_{1}\right)}{\sqrt{\operatorname{var} X_{i} \operatorname{var} X_{1}}} \\
\text { Soit } I_{2,3, \ldots, n}=\int_{h_{2}}^{\infty} \ldots \int_{h_{n}}^{\infty} f_{3}\left(u_{2}, \ldots, u_{n}\right) d_{2} \ldots d u_{n}
\end{gathered}
$$

où $f_{3}\left(u_{2}, \ldots, u_{n}\right)$ est la densité de la loi multinormale centrée réduite dont la matrice des corrélations est la matrice des corrélations partielles entre $X_{2}, \ldots, X_{n}$ sachant $X_{1}$.

Les principales difficultés du calcul de $\Delta \mathrm{G}_{\mathrm{y}}$ sont par conséquent le calcul numérique de l'intégrale multiple d'une loi multinormale tronquée et son opération inverse (recherche des points de troncature connaissant la valeur de l'intégrale). Un certain nombre 
de techniques d'évaluation de telles intégrales ont été proposées (voir en particulier JAIN \& AMBLE, 1962 ; et bibliographie de GuPTA, 1963), mais sont généralement inadaptées ou extrêmement lourdes dès que l'on dépasse la dimension 2.

Milton (1972) a proposé une technique d'évaluation assez générale qu'il aurait été éventuellement possible d'utiliser dans cette étude, mais nous avons préféré une méthode originale et bien adaptée, proposée par DuTT $(1973,1975)$ et DuTT \& SOMS (1976), méthode nettement plus rapide à précision égale que celle de MiLton, selon DuTT lui-même (1973). Les algorithmes ayant permis l'écriture du programme de calcul (en langage APL) sont résumés en annexe. Il est à souligner que par cette dernière méthode, il est tout à fait concevable d'envisager le calcul d'intégrales de dimension assez élevée (6 ou 7). Enfin, la recherche des points de troncature à partir des $\alpha_{i}$ est résolue par itérations successives, à l'aide d'un algorithme faisant intervenir les dérivées première et seconde des intégrales considérées.

\section{F. Choix des reproducteurs}

Les principales modalités de sélection pour chacune des 4 voies de transmission du progrès génétique sont résumées dans le tableau 2 .

\section{Voie père-fille}

En France, la capacité de testage (p. 100 de femelles contrôlées et d'ascendance connue) constitue classiquement le facteur limitant de la mise à l'épreuve de taureaux sur descendance. Aussi a-t-on considéré que cette capacité de testage reste fixe, quel que soit le schéma envisagé. Après un éventuel contrôle individuel en station puis un contrôle de spermatogénèse, 50 taureaux sont mis en testage, puis sont sélectionnés à l'âge de 6 ans sur la production laitière (et éventuellement le pointage laitier) de leurs filles.

L'index caractérisant la valeur génétique laitière des taureaux qui a été utilisé est supposé conforme à la théorie des index : il est non biaisé et en particulier tient compte de la date de naissance du taureau, en présence de progrès génétique dans la population. En conséquence, l'existence d'un progrès génétique laitier annuel $\Delta G_{L}$ induit un décalage des distributions des valeurs génétiques estimées de séries de testage successives. La procédure optimale de sélection est alors une troncature unique sur l'ensemble des distributions : ne sont retenus que les taureaux dont l'index est supérieur à une valeur donnée indépendante de l'âge du taureau (BICHARD, 1972).

\section{Voie père-fils}

Parmi les 50 taureaux testés, 3 sélectionnés sur la production laitière (et éventuellement le pointage laitier) de leurs filles, sont utilisés comme pères à taureau pendant 2 ans. Les effectifs d'animaux candidats à la sélection et sélectionnés étant faibles, l'espérance de la valeur génétique des animaux retenus est en fait surestimée, si elle est calculée à partir des distributions tronquées portant sur une population infinie. L'extension au cas d'une sélection en plusieurs étapes de l'approximation du facteur correctif tenant compte de l'échantillonnage de la population (BURRows, 1972) est probablement possible, mais n'a pas été retenue pour ne pas surcharger les calculs, et parce qu'il nous a semblé que le gain relativement faible de précision n'affecterait certainement pas le sens des conclusions de cette étude. 


\section{TABLEAU 2}

Schéma de référence: hypothèses concernant le choix des reproducteurs mâles et femelles. Reference scheme : assumptions about the choice of AI sires and dams.

Voir père-fille

- Nombre de taureaux mis en testage sur descendance, par an ........

- Nombre moyen de premières lactations connues de filles de taureau de testage

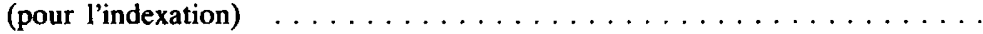

- Elimination en station pour d'autres critères que ceux étudiés (spermatogénèse,

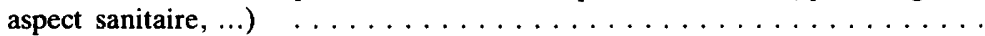

- Stock de semence disponible au moment de l'indexation $\ldots \ldots \ldots \ldots \ldots$

- Age à l'indexation .......................

- Nombre maximal de doses utilisées/taureau/an $\ldots \ldots \ldots \ldots \ldots \ldots$

- Durée maximale d'utilisation de la semence après indexation $\ldots \ldots \ldots \ldots$

- Durée maximale de prélèvement des taureaux après indexation . . . . .

- Quantité moyenne prélevée par taureau et par an après indexation . . . .

- Besoin annuel en doses pour toute la population : $400000 \times 1,7 \ldots \ldots$.

Voie père-fils

- Nombre de pères à taureau retenus par an $\ldots \ldots \ldots \ldots \ldots \ldots$

- Durée moyenne d'utilisation des pères à taureau

\section{Voie mère-fille}

- Sélection massale des femelles : pourcentage des éliminations dues à un niveau

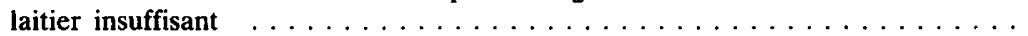

- Sélection sur ascendance : pourcentage de génisses de renouvellement conservées sur les index sur ascendance :

- parmi les génisses dont les mères ont 1 lactation connue ou plus

- parmi les génisses dont les mères n'ont pas encore de lactations connues (primipares)

le reste pour assurer le renouvellement soit :

Voie mère-fils

- Nombre de mères à taureau nécessaires par taurillon entrant en station . .

- Nombre de lactations connues nécessaires pour être mère à taureau . . . . .

* Valeur obtenue de façon à satisfaire les besoins en génisses de renouvellement $(26000)$ en tenant compte du sex ratio à la naissance, du taux de mortalité avant l'âge de 2 ans et du pourcentage de génisses issues des $74000(100000-26000)$ mères ayant au moins une lactation connue.

\section{Voie mère-fille}

On a considéré que la sélection sur cette voie ne portait que sur la production laitière : les génisses de renouvellement sont les filles des vaches ayant les meilleurs index femelles (sélection sur ascendance). Les vaches en lactation sont soumises à une 
sélection sur performances propres. Faute de données précises en la matière, on a retenu qu'un tiers des éliminations entre la $1^{\text {re }}$ et la $2^{e}$ lactation et entre la $2^{e}$ et la $3^{\text {e }}$ lactation est lié à un index laitier insuffisant. L'index utilisé combine l'information sur ascendance et sur les lactations réalisées. Il a été supposé de la forme $I_{n}=I_{a}+b_{n}\left(I_{a}-P\right)$ où $I_{a}$ est l'index sur ascendance, $P$ la moyenne des lactations corrigées déjà réalisées et $b_{n}$ le coefficient de régression du résidu $I_{a}-P$ sur $I_{n}$.

\section{Voie mère-fils}

Les mères à taureau sont sélectionnées parmi les vaches ayant réalisé au moins 3 lactations. L'intensité de sélection sur la production laitière est déterminée par le nombre moyen de mères à taureau potentielles nécessaire pour obtenir effectivement un taurillon entrant en station. Ce paramètre est sous la dépendance de nombreux facteurs (sex-ratio, contraintes sanitaires imposées concernant le veau, la mère ou le troupeau d'origine, possibilités de transplantation embryonnaire, ainsi que celles liées à la recherche pas toujours exhaustive des candidates mères à taureau).

La valeur retenue a été de 6 mères à taureau/taurillon (LINDHE, 1968), sauf indication contraire, une gamme de valeurs allant de 0,5 (fort taux de transplantation) à 15 mères à taureau/taurillon ayant été étudiée.

Là encore, on a supposé qu'il y avait sélection des mères appartenant à différentes cohortes par troncature unique. Ne sont retenues comme mères à taureau que les vaches dont les valeurs génétiques estimées pour tous les caractères sélectionnés sont supérieurs à des seuils indépendants de l'âge des animaux.

\section{Résultats}

\section{A. Progrès génétique laitier " maximum "}

Il est obtenu lorsqu'aucune sélection sur des caractères secondaires n'est réalisée (tabl. 3). Avec les hypothèses présentées aux tableaux 1 et 2 , le progrès génétique annuel (de RENDEL \& ROBERTSON) est de 0,192 écart-type génétique de la production laitière $\left(\sigma_{\mathrm{G}}\right.$ lait) soit $96 \mathrm{~kg}$ avec $\sigma_{\mathrm{G}}=500 \mathrm{~kg}$. C'est bien entendu une valeur très optimiste compte tenu des hypothèses très favorables envisagées.

Les gains génétiques sur la production laitière cumulés à l'infini et actualisés à un taux de $10 \%$ sont de 1,66 écart-type génétique, soit environ 9 ans de gains sans actualisation.

Comme aucune sélection n'est réalisée sur les caractères secondạires, l'évolution des niveaux génétiques de ceux-ci est directement proportionnelle à celui de la production laitière : si la corrélation génétique entre celle-ci et un caractère tel que, par exemple, la conformation bouchère est de $-0,30$, la perte génétique annuelle sur ce caractère sera de $0,3 \times 0,192=0,0576$ écart-type génétique (soit près de $0,6 \mathrm{p}$. 100/an pour un coefficient de variation de 10 p. 100). 


\section{TABLEAU 3}

Progrès génétique laitier maximum (schéma de sélection sans aucune sélection sur des caractères secondaires; avec les hypothèses exposées aux tableaux 1 à 2).

Maximum genetic gain for milk production (selection scheme without selection on secondary traits, with the assumption shown in tables 1 to 2).

\begin{tabular}{|c|c|c|c|c|}
\hline Voie & $\begin{array}{l}\text { Intervalle } \\
\text { de génération } \\
\text { (en année) }\end{array}$ & $\begin{array}{l}\text { Supériorité } \\
\text { génétique } \\
\text { moyenne des } \\
\text { reproducteurs } \\
\text { (en écart-type } \\
\text { génétique) }\end{array}$ & $\begin{array}{l}\text { Progrès génétique } \\
\text { laitier annuel } \\
\text { (en écart-type } \\
\text { génétique) }\end{array}$ & $\begin{array}{l}\text { Contribution } \\
\text { relative au } \\
\text { progrès annuel }\end{array}$ \\
\hline Père-fils $\ldots$. & 7,5 & 1,74 & 0,232 & 31 p. 100 \\
\hline Père-fille $\quad \ldots$ & 7,5 & 1,17 & 0,156 & 21 p. 100 \\
\hline Mère-fils & 6,6 & 1,88 & 0,285 & 38 p. 100 \\
\hline Mère-fille & 5,6 & 0,43 & 0,077 & 10 p. 100 \\
\hline Moyenne & 6,8 & 1,305 & 0,192 & 100 p. 100 \\
\hline
\end{tabular}

\section{B. Dispersion des objectifs de sélection}

Un grand nombre d'études concernant les liaisons entre la production laitière et la vitesse de croissance intra-race (LEBBE, 1978) d'une part et entre la production laitière et la conformation laitière (LE MEZEC, 1980 ; COLLEAU, 1982a) d'autre part semblent indiquer que les corrélations génétiques entre ces caractères sont proches de 0 . Dans un premier temps, nous avons donc considéré la situation simple où les caractères secondaires envisagés sont indépendants de la production laitière. Dans ce cas, les pertes de progrès génétique laitier résultent uniquement de la dispersion des objectifs de sélection.

\section{Sélection sur la vitesse de croissance}

Le nombre de taureaux mis à l'épreuve sur descendance chaque année étant supposé constant, cette sélection, réalisée sur performance propre contrôlée en station, conduit à un accroissement du nombre de jeunes taurillons (et donc du nombre de mères à taureau) à rechercher : seule la voie mère-fils sera affectée par cette sélection.

La chute de progrès génétique laitier, par rapport au schéma ne s'intéressant qu'à la production laitière décrite précédemment, est présentée à la figure 1 . Cette chute n'est pas du tout linéaire. Elle est faible pour les taux d'élimination en sortie de station assez bas (inférieur à 2 p. 100 pour une élimination de 30 p. 100 des animaux). Mais le gain annuel sur la vitesse de croissance est alors lui aussi relativement modeste (inférieur à 0,25 p. 100 par an pour un coefficient de variation de 10 p. 100).

Par contre, pour les fortes pressions de sélection nécessaires à l'obtention d'un gain assez important sur la vitesse de croissance (élimination de 2 taurillons sur 3 pour $\Delta \mathrm{G}_{\mathrm{a}} \simeq 0,05 \sigma$ ), la diminution du progrès génétique laitier devient assez sensible (plus de 5 p. 100), conséquence d'une réduction nette de la contribution de la voie mère-fils à l'amélioration du caractère principal. 


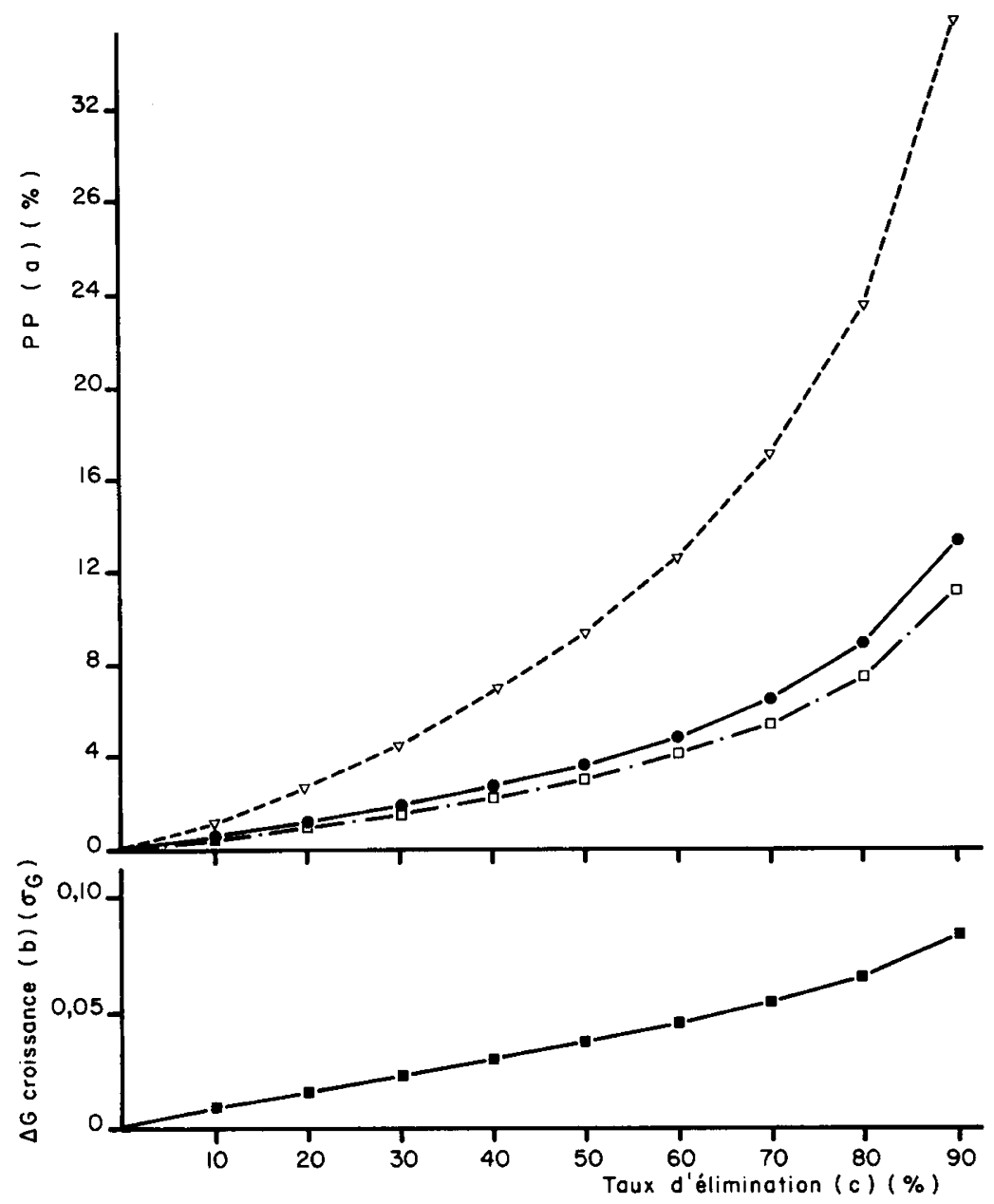

FIGURE 1

Perte de progrès génétique laitier due à une sélection sur la vitesse de croissance (corrélation nulle avec la production laitière), par rapport au schéma laitier pur " maximaliste".

Loss in genetic gain for milk production due to selection on growth rate

(null correlation with milk yield), as compared to the pure dairy scheme.

(a) $\nabla----\nabla$ ( Pourcentage de perte sur le progrès génétique $\left(\mathrm{PP}_{\mathrm{G}}\right)$ de la voie mère-fils. Loss (per cent) in genetic gain $\left(P P_{G}\right)$ via the bull-dam path.

$\longrightarrow$ Pourcentage de perte sur le progrès génétique annuel $\left(\mathrm{PP}_{\mathrm{G}}\right)$.

Loss (per cent) in annual genetic gain $\left(\mathrm{PP}_{\mathrm{G}}\right)$.

$\square--\square \quad$ ( Pourcentage de perte sur le cumul actualisé des gains génétiques sur la production laitière $\left(\mathrm{PP}_{\mathrm{R}}\right)$.

Loss (per cent) in accumulated discounted genetic gains $\left(P P_{R}\right)$ for milk production.

(b) $\mathbf{\square}$ Gain génétique correspondant sur la vitesse de croissance (en écart-type génétique).

Corresponding genetic gain for growth rate (in genetic standard deviation).

(c) (Pourcentage de taurillons éliminés en station sur la vitesse de croissance.

Percentage of bulls culled after growth rate performance test. 
Afin de mettre en évidence une éventuelle influence de l'effort initial de sélection laitière sur les résultats, on a fait varier l'efficacité du schéma de référence en partant d'hypothèses nettement moins optimistes quant aux pressions de sélection effectivement réalisées sur la production laitière pour chacune des 4 voies de transmission des gènes (situations II et III du tableau 4; avec cette fois pour les 3 situations envisagées, élimination de 25 p. 100 des mères à taureau sur la conformation laitière). On constate que pour un même pourcentage éliminé en sortie de station, "l'efficacité laitière " de la voie mère-fils chute d'autant plus rapidement que la sélection sur la production laitière du schéma de référence est moins intense (schémas II et III). Si l'impact de la sélection du caractère secondaire est presque semblable pour les 3 situations, lorsque la pression de sélection correspondante est faible, il est par contre assez nettement différent, surtout en valeur relative, pour les fortes pressions : ainsi, lorsque 75 p. 100 des animaux sont éliminés en sortie de station, le niveau génétique laitier moyen des mères à taureau chute de 22 p. 100 dans la situation I et de 30 p. 100 dans la situation III par rapport à ce qu'il était en l'absence de sélection sur le caractère secondaire.

\section{TABLEAU 4}

Conséquences d'une sélection sur la vitesse de croissance, en fonction de l'importance initiale accordée à la sélection laitière.

Consequences of a selection for growth rate,

according to the previous intensity of selection on milk yield.

\begin{tabular}{|c|c|c|c|c|c|c|}
\hline \multirow{2}{*}{$\begin{array}{l}\text { Elimination } \\
\text { sur vitesse } \\
\text { de croissance } \\
\text { en station }\end{array}$} & \multicolumn{3}{|c|}{$\begin{array}{l}\text { Perte de progrès génétique laitier } \\
\text { sur la voie mère-fils (en écart-type) } \\
\text { selon les situations initiales }\end{array}$} & \multicolumn{3}{|c|}{$\begin{array}{l}\text { Pourcentage de perte correspondant } \\
\text { sur le progrès génétique annuel global }\end{array}$} \\
\hline & I & II & III & I & II & III \\
\hline 12,5 p. 100 & 0,035 & 0,039 & 0,042 & 0,7 & 1,0 & 1,1 \\
\hline 25 p. 100 & 0,070 & 0,080 & 0,086 & 1,5 & 2,1 & 2,3 \\
\hline 50 p. 100 & 0,182 & 0,202 & 0,219 & 3,7 & 4,8 & 6,0 \\
\hline 75 p. 100 & 0,388 & 0,433 & 0,464 & 7,9 & 11,0 & 12,9 \\
\hline
\end{tabular}

Situations initiales (sans sélection sur la vitesse de croissance) :

I : schéma décrit aux tableaux 1 et 2 (identique à celui de la fig. 1) + élimination de $25 \mathrm{p} .100$ des mères à taureau sur la conformation laitière.

II : identique au I mais :

- pas de sélection sur la voie mère-fille ;

- 10 mères à taureau/taurillon entrant en station ;

- 6 pères à taureau utilisés pendant 3 ans ;

$-1 / 3$ des taureaux retenus à l'indexation.

III : identique au II mais :

- 15 mères à taureau/taurillon entrant en station ;

$-1 / 2$ des taureaux retenus à l'indexation. 


\section{Sélection sur la conformation laitière}

Les répercussions sur le progrès génétique laitier d'une sélection sur le pointage laitier pour la voie mère-fils seule sont très naturellement tout à fait semblables à ce qu'elles étaient pour la vitesse de croissance. Le progrès génétique réalisé sur le pointage est malgré tout plus faible, car l'héritabilité de ce caractère est elle-même plus faible. Dès lors, on est tenté d'étudier l'effet d'une sélection simultanée du caractère secondaire sur la voie mère-fils et sur l'une des voies mâles (ou les 2).

La figure 2 illustre les conséquences d'une telle sélection pour des pressions de sélection croissantes sur chacune des 3 voies (en supposant néanmoins dans tous les cas une élimination minimale d'une vache sur 4 sur la voie mère-fils).

Il apparaît très clairement qu'un même progrès génétique sur le caractère secondaire peut être obtenu en répartissant les efforts de sélection de façon différente selon les 3 voies. La perte laitière correspondante peut alors varier dans une large gamme de

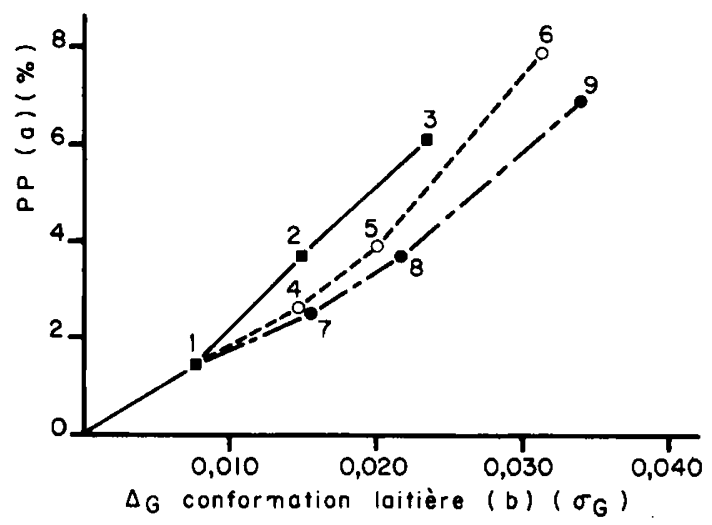

FIGURE 2

Perte de progrès génétique laitier correspondant à un progrès génétique donné sur le pointage laitier.

Loss in genetic gain for milk production due to a given genetic gain on dairy type.

(a) Pourcentage de perte sur le progrès génétique laitier annuel.

Loss in per cent in annual genetic gain for milk yield.

(b) Progrès génétique annuel sur le pointage laitier (en unités d'écart-type génétique).

Annual genetic gain for type (in genetic standard deviation).

( Pression de sélection croissante sur la voie mère-fils

(élimination : $25,50,75$ p. $100:$ points $1,2,3$ ).

Increasing selection of bull dams.

------O Elimination de 25 p. 100 des mères à taureau puis pression de sélection croissante sur la voie père-fille.

(élimination : $12,5,25,50$ p. 100 : points $4,5,6$ ).

Culling of $25 \mathrm{p} .100$ of bull dams and increasing selection of cow sires.

--- Elimination de 25 p. 100 des mères à taureau puis pression de sélection croissante sur la voie père-fils.

(élimination : $12,5,25,50$ p. 100 : points $7,8,9$ ).

Culling of 25 p. 100 of bull dams and increasing selection of bull sires. 
valeurs. D'un point de vue génétique, la voie mère-fils paraît être la moins efficace car les candidates à la sélection des mères à taureau sont connues avec une précision beaucoup plus faible sur le caractère secondaire que les mâles après testage sur descendance.

\section{Réponse corrélée}

La perte de progrès génétique laitier peut être entraînée non seulement par un effet de dispersion des objectifs mais aussi par réponse génétique indirecte défavorable, conséquence d'une liaison non nulle entre caractères principal et secondaire.

\section{Sélection sur la conformation bouchère}

Si l'opposition production de lait - production de viande entre race ne fait pratiquement aucun doute, elle semble beaucoup moins évidente intra-race (LEBBE, 1978). Néanmoins, un certain nombre d'auteurs considèrent qu'il existe une corrélation génétique négative entre la production laitière et les critères caractérisant la conformation bouchère des animaux (MAŚNon, 1964, 1972 ; LANGHOLZ et al., 1972 cités par LEBBE, 1978 ; Colleau, 1982b).

A cause de l'imprécision avec laquelle sont connues ces corrélations et de la diversité des critères de sélection possibles, on a étudié l'évolution simultanée des progrès génétiques annuels « lait » et "viande » pour des taux d'élimination croissants sur le critère boucher (mesuré en station) pour 3 valeurs de corrélations génétiques : $0,-0,15$, - 0,30 (fig. 3). La situation de référence correspond toujours au schéma ne s'intéressant qu'à l'amélioration de la production laitière.

Quelle que soit la valeur de corrélation envisagée, les niveaux génétiques du critère boucher évoluent de façon très semblable lorsque l'effort de sélection sur ce caractère s'accroît. La signification de ces niveaux n'est cependant pas du tout la même dans chacun des 3 cas. Rappelons qu'en absence de sélection en station et pour un coefficient de variation de $10 \mathrm{p}$. 100 , la réponse corrélée à la forte sélection laitière entraîne une détérioration annuelle de près de 0,3 p. 100 (pour $r_{G}=-0,15$ ) et de près de 0,6 p. 100 (pour $r_{G}=-0,30$ ) de la conformation bouchère. Dans ces conditions, il est nécessaire d'éliminer plus d'un animal sur 3 (pour $r_{G}=-0,15$ ) et près de 2 animaux sụr 3 (pour $r_{G}=-0,30$ ) sur la conformation bouchère pour simplement maintenir le niveau génétique de ce caractère ! Parallèlement, la chute du progrès génétique laitier est nettement accrue par rapport au cas où les caractères sont indépendants, la différence entre ces 2 situations devenant importante lorsque la pression de sélection sur le critère «viande » est élevée (réponse corrélée très défavorable).

Une étude plus détaillée fait apparaître une évolution génétique de ce critère plus défavorable sur la voie père-fils que sur la voie père-fille (le plus fort niveau génétique laitier correspondant entraîne une plus importante dégradation de la conformation bouchère). Par contre, l'impact de la sélection sur le caractère secondaire mesuré par le pourcentage de perte sur le lait défini ci-dessus, est toujours plus faible sur la voie père-fils. Là encore, la pression de sélection laitière nettement plus forte limite les répercussions de la dispersion des objectifs de sélection (cf. III B1). Mais la voie la plus touchée reste la voie mère-fils : le besoin plus important en mères à taureau entraîne une forte diminution de la pression de sélection sur la production laitière. 

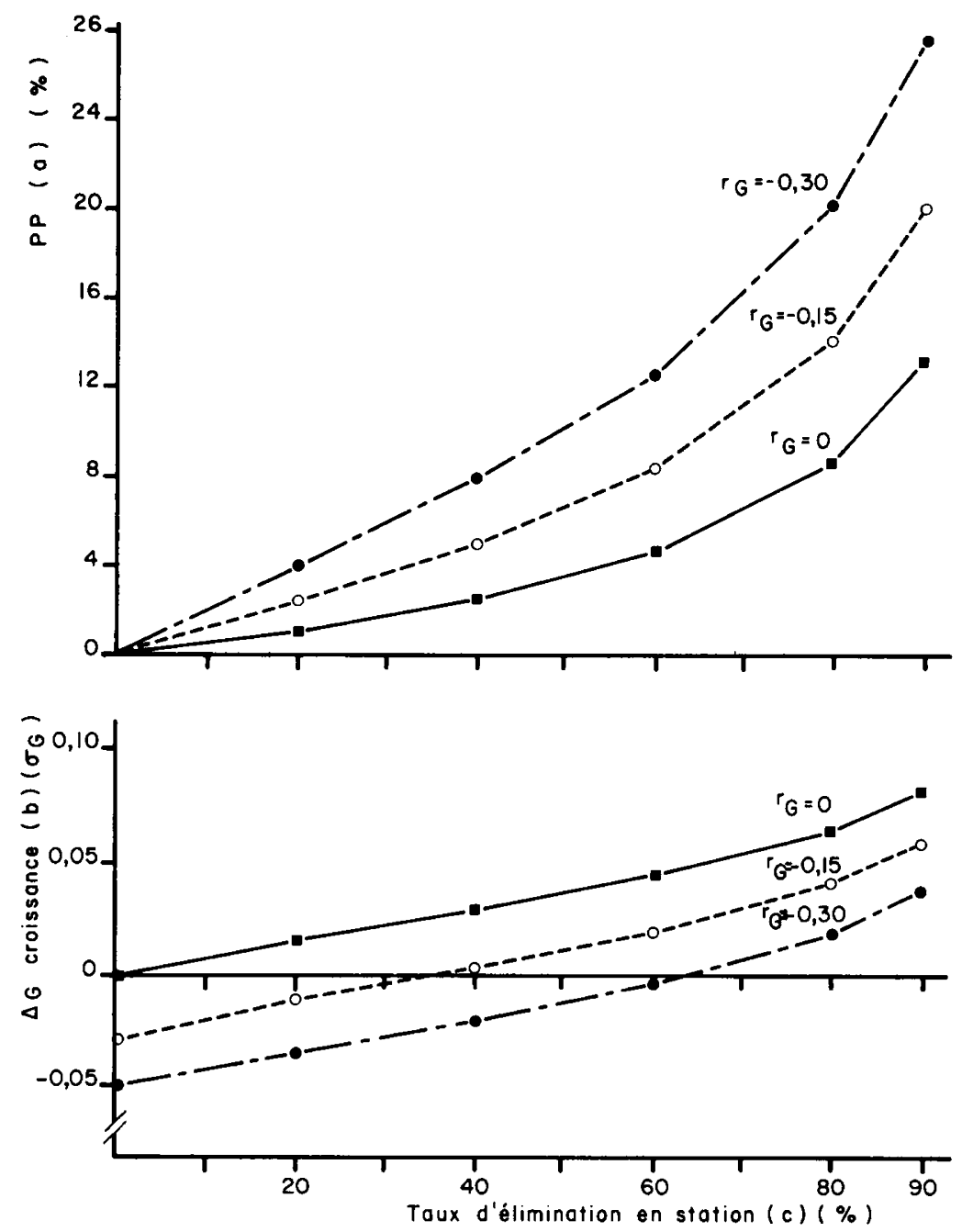

Figure 3

Conséquence d'une sélection sur un critère de production de viande corrélé négativement à la production laitière.

Consequence of selection on a beef trait negatively correlated with milk yield.

(a) Pourcentage de perte sur le progrès génétique laitier annuel. Loss in per cent in annual genetic gain for milk yield.

(b) Gain génétique correspondant sur la vitesse de croissance (en écart-type génétique). Corresponding genetic gain for growth rate (in genetic standart deviation).

(c) Pourcentage de taurillons éliminés en station sur la vitesse de croissance. Percentage of bulls culled after growth rate performance test. 
Pour atténuer cette diminution, on peut envisager d'accroître l'efficacité de la voie mère-fille en utilisant, par exemple, massivement les techniques de transplantation embryonnaire. On ne cherche plus dans ce cas à accroître la pression de sélection laitière (approche classique) mais à disposer d'un plus grand nombre de taurillons à tester sur performances propres, sans réduire simultanément la pression de sélection laitière des mères à taureau. La perte sur le progrès génétique laitier n'est alors plus liée à la dispersion des objectifs de sélection mais à la seule réponse corrélée à la sélection.

Pour une corrélation génétique entre la production de lait et celle de viande de $-0,15$ et en faisant passer de 6 à seulement 4 le nombre de mères à taureau retenues par taurillon entrant en station, on peut maintenir le niveau génétique sur le critère de production de viande avec une perte de seulement 2,1 p. 100 sur le progrès laitier (contre 5 p. 100 dans le cas d'une sélection sans accroissement de l'efficacité de la voie mère-fils).

\section{Sélection sur la conformation laitière}

Nous avons vu précédemment (III B2) qu'un même progrès génétique sur la conformation laitière pouvait être obtenu pour des modalités assez différentes de répartition des efforts de sélection sur les voies père-fils, père-fille et mère-fils. On peut par conséquent rechercher les combinaisons des pressions de sélection sur ces 3 voies qui affectent le moins la production laitière, pour un progrès génétique donné sur le pointage laitier. Cette recherche a été effectuée pour 2 valeurs de corrélation génétique entre la production et la conformation laitière $(-0,10$ et $+0,20)$ reflétant approximativement la diversité des estimations couramment citées (LE MEZEC, 1980 ; ColleAu et al., 1982a). Pour couvrir une gamme assez étendue de valeurs de progrès génétique sur le pointage laitier, toutes les combinaisons de 5 pressions de sélection différentes sur chacune des 3 voies ont été étudiées (voie mère-fils : élimination de $0,12,5,25,50$ ou 75 p. 100 des animaux sur la conformation laitière; voies père-fils et père-fille : élimination de $0,12,5,25,33$ ou 50 p. 100 des animaux après contrôle sur descendance et avant sélection sur la production laitière). On a regroupé au tableau 5 les résultats de quelques-unes de ces modalités de sélection, extrêmes quant à leur répercussion sur le progrès génétique laitier, mais conduisant à un même progrès annuel sur la conformation laitière.

L'influence du signe de la corrélation génétique entre caractères principal et secondaire est particulièrement nette, même compte tenu des faibles valeurs absolues considérées : dans un cas $\left(\mathrm{r}_{\mathrm{G}}=+0,20\right)$, l'évolution du pointage laitier est très favorable, même en l'absence de sélection. Dans l'autre $\left(\mathrm{r}_{\mathrm{G}}=-0,10\right)$, l'effort à consentir pour simplement maintenir la conformation laitière n'est pas négligeable.

Ce tableau 5 souligne, de plus, l'importante diversité des combinaisons conduisant à un même progrès génétique annuel. On peut, par exemple (pour le schéma « favorable $»$ ), obtenir un progrès annuel de 0,06 écart-type génétique en portant indifféremment l'essentiel de la sélection sur l'une ou l'autre des 3 voies considérées. Mais les pertes sur le progrès génétique laitier (annuel ou cumulé), dues à la sélection sur la conformation laitière, suivent cette même diversité. Les valeurs absolue et surtout relative de ces pertes dépendent très fortement, là encore, du signe des corrélations génétiques entre caractère principal et secondaire. Très conséquentes pour $r_{G}=-0,10$, elles sont par contre beaucoup plus faibles pour $r_{G}=+0,20$. 


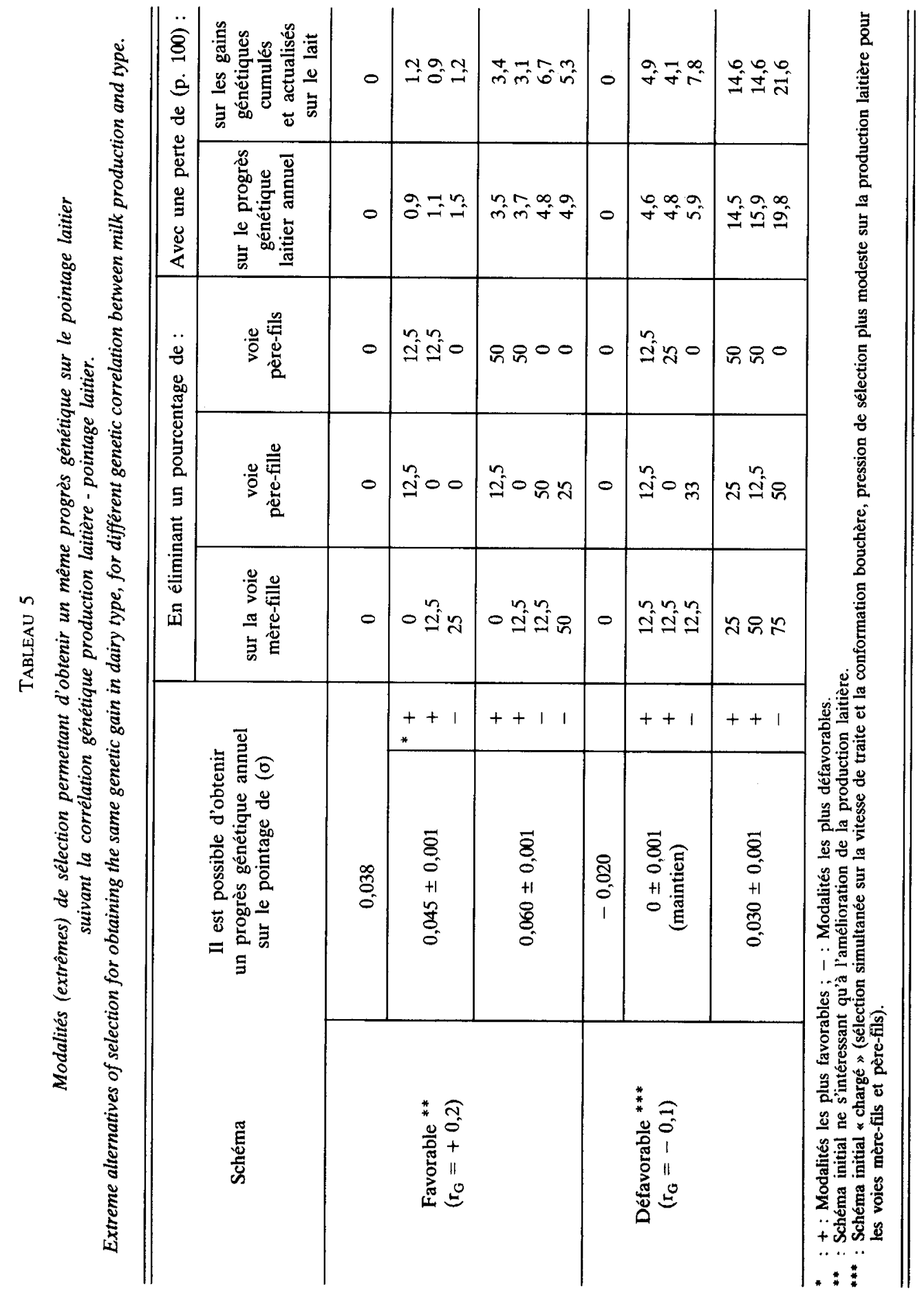


On peut d'autre part remarquer que les combinaisons conduisant aux pertes les plus faibles (optimum génétique et non nécessairement économique !) sont celles pour lesquelles l'effort de sélection est relativement bien réparti selon les 3 voies : une sélection du caractère secondaire sur une seule des 3 voies aura un impact plus négatif sur le progrès génétique laitier.

Enfin, le classement des différentes combinaisons est légèrement différent lorsque l'on considère comme critère de comparaison le cumul des gains génétiques actualisés. En particulier, toutes les combinaisons pour lesquelles la pression de sélection sur la conformation laitière sur la voie père-fille est assez forte sont très défavorables pour ce critère. Les répercussions sur la production laitière de la sélection du caractère secondaire se font sentir beaucoup plus tôt sur la population commerciale, ce qui est pris en compte par l'actualisation.

\section{Discussion}

Il convient de noter, tout d'abord, que la modélisation de la sélection envisagée nous a conduit à faire des simplifications dont certaines peuvent paraître fortes. Parmi celles-ci, on peut rappeler que l'on a considéré une population fermée dont les paramètres démographiques ne variaient pas avec le temps. On a négligé l'influence de la sélection sur les variances et corrélations entre caractères de même que l'augmentation du taux de consanguinité. L'hypothèse d'une sélection à niveaux indépendants ne correspond que très grossièrement à la réalité. Le modèle génétique de base a été supposé vérifié pour tous les niveaux de performance, en particulier pour les mères à taureau. Enfin, on n'a pas tenu compte de la réduction d'intensité de sélection de la voie père-fils occasionnée par la petite taille des populations concernées.

Compte tenu de ces restrictions, les calculs réalisés ont permis de mettre en évidence une perte du progrès génétique laitier due à la sélection d'un caractère secondaire variant couramment entre 1,5 et 15 p. 100 suivant le contexte et les pressions de sélection appliquées. En se limitant aux seuls caractères génétiquement indépendants de la production laitière, cette perte peut aller jusqu'à 6 p. 100 (respectivement 13 p. 100) pour une élimination d'un animal sur 2 (respectivement 3 sur 4) en sortie de station (cas $\mathrm{n}^{\circ}$ III du tableau 4). La sélection du caractère secondaire ne concernant généralement pas l'ensemble des 4 voies de transmission des gènes, ces valeurs cachent en fait une dégradation du niveau laitier beaucoup plus importante sur certaines de ces voies.

La réduction de l'intensité de sélection sur la production laitière, jusqu'à présent considérée comme faible lorsque caractères principal et secondaire sont indépendants (LiNDHE, 1968 ; HiNKs, 1970b ; HiLL, 1971) est donc loin d'être négligeable.

D'autre part, on a pu montrer que cette perte de progrès génétique laitier est d'autant plus importante :

1 - que le progrès génétique recherché sur le caractère secondaire est plus élevé.

$\mathrm{Si}$ la sélection de ce caractère s'exerce sur une seule voie (ou de façon identique sur plusieurs voies : exemple des 2 voies mâles pour la sélection après contrôle individuel), cette perte peut être calculée grossièrement à partir d'une expression de la forme :

$$
\Delta G_{L}-\Delta G_{L_{0}}=a\left(\Delta G_{S}-\Delta G_{\text {So }}\right)^{b}
$$


où $-\Delta \mathrm{G}_{\mathrm{L}}$ et $\Delta \mathrm{G}_{\mathrm{S}}$ sont les progrès génétiques annuels globaux (en écart-type génétique) sur la production laitière et le caractère secondaire.

- $\Delta \mathrm{G}_{\mathrm{Lo}}$ et $\Delta \mathrm{G}_{\mathrm{So}}$ sont les valeurs correspondantes en l'absence de sélection sur le caractère secondaire $\left(\Delta \mathrm{G}_{\mathrm{So}}=0\right.$ si les caractères sont indépendants).

- $a$ et $b$ sont des coefficients dont les valeurs dépendent du contexte (corrélations génétiques, intensité de sélection laitière, etc.).

A titre d'exemple, pour la sélection sur la vitesse de croissance en station dans les conditions de la figure 3 , on a :

$$
\begin{array}{llll}
\mathrm{a}=-0,900 & \mathrm{~b}=1,45 & \Delta \mathrm{G}_{\mathrm{So}}=0 & \text { pour } \mathrm{r}_{\mathrm{G}}=0 \\
\mathrm{a}=-0,944 & \mathrm{~b}=1,23 & \Delta \mathrm{G}_{\text {So }}=-0,055 \text { pour } \mathrm{r}_{\mathrm{G}}=-0,3
\end{array}
$$

et pour une sélection sur le pointage laitier sur la voie mère-fils seule (conditions de la fig. 2, $\left.\mathrm{r}_{\mathrm{G}}=0\right): \mathrm{a}=-1,546 \quad \mathrm{~b}=1,30 \quad \Delta \mathrm{G}_{\mathrm{So}}=0$.

2 - que le schéma considéré est globalement peu intensif en ce qui concerne la sélection sur la production laitière, pour des raisons d'organisation par exemple.

En conséquence, la sélection sur des caractères secondaires sera d'autant moins coûteuse que le schéma dans lequel elle s'intègre est plus rationnel et efficace.

3 - que certaines voies de sélection sont peu intensives sur la production laitière parce que tout l'effort de sélection du (ou des) caractère (s) secondaire (s) y est reporté.

Lorsque cela est possible, il convient donc de bien répartir cet effort sur plusieurs (voire l'ensemble) de ces voies.

4 - que les caractères secondaires sont défavorablement liés à la production laitière.

La sensibilité des résultats obtenus aux valeurs et surtout au signe des corrélations génétiques est importante. Lorsqu'il y a opposition entre caractères, même faible $\left(r_{G}=-0,10\right.$ (tableau 5), $r_{G}=-0,15$ (fig. 3)), l'intensité de sélection élevée sur la production laitière limite fortement les possibilités d'une évolution favorable du caractère secondaire sauf pour d'assez fortes pressions de sélection sur celui-ci, dont l'impact négatif sur le progrès laitier se fait alors très nettement ressentir. Aussi paraît-il nécessaire d'intégrer ce point dans les études de rentabilité économique des schémas de sélection comme l'ont fait déjà Brascamp (1974) et Cunningham \& Mc Clintock (1974). L'hypothèse simplificatrice d'indépendance entre caractères généralement retenue dans ce genre d'étude apparaît en effet comme relativement forte.

\section{Conclusion}

La technique de DuTt pour le calcul numérique des intégrales de loi multinormale utilisée ici permet donc d'envisager l'étude de la sélection multicaractères sans les classiques limitations pratiques quant au nombre d'étapes ou de critères de sélection retenus et quelles que soient les corrélations génétiques entre ces caractères.

Elle nous a permis de mettre en évidence le rôle important de la pression de sélection laitière sur l'ampleur des répercussions sur le progrès génétique laitier de la sélection d'un caractère secondaire. 
Il apparaît également que l'on peut limiter ces répercussions par une répartition judicieuse des efforts de sélection selon les différentes voies de transmission du patrimoine génétique d'une génération à la suivante.

Enfin, il convient de mettre l'accent sur la nécessité d'une bonne connaissance des corrélations génétiques entre caractères sélectionnés. Elle peut en effet conditionner fortement le résultat des études de rentabilité des schémas de sélection. Ce dernier point, qui s'ajoute aux difficultés de calcul des pondérations économiques à accorder à des caractères très différents et quelquefois subjectifs (ELSEN, 1977) ne rend que plus délicat le problème de l'évaluation objective de l'intérêt de la sélection des caractères secondaires chez les bovins laitiers.

Bien que ne faisant appel à aucun critère économique synthétique permettant de comparer les différentes situations envisagées, cette étude permet en définitive de confirmer la priorité absolue qu'il y a, dans les schémas de sélection des bovins laitiers, à choisir les caractères secondaires parmi ceux qui ont un intérêt économique prouvé. Pour compenser de manière avantageuse la baisse de progrès génétique engendrée par une dispersion des objectifs de sélection et/ou une opposition même très faible des caractères sélectionnés, il apparaît impératif, en effet, que l'importance économique du caractère secondaire ne puisse être mise en doute.

\section{Remerciements}

Nous tenons à remercier J.J. Colleau pour son importante contribution à la réalisation de ce travail ainsi que J.C. Mocovot et J.M. ELSEN. Nous remercions par ailleurs les lecteurs anonymes mandatés par la revue, pour leurs utiles critiques et suggestions.

Reçu le 2 septembre 1983.

Accepté le 2 avril 1984.

\section{Références bibliographiques}

Bichard M., Pease H.R., Swales P.H., Ozkutuk K., 1972. Selection in a population with overlapping generations. Anim. Prod., 17, 215-227.

Booth A.D., 1957. Numerical methods. 195 pp., Butterworths Scientific Public, London.

BRASCAMP E.W., 1974. Model calculations concerning economic optimalization of AI. Breeding with cattle. III. Profitability of performance testing in a dual-purpose breed according to meat production and the effect of beed crossing. Z. Tierz. Zuchtgsbiol., 91, 176-187.

Burrows P.M., 1972. Expected selection differentials for directional selection. Biometrics, 28, 1091-1100.

Cochran W.G., 1951. Improvement by means of selection. Proceedings of the second Berkeley Symposium Mathematical Statistics and Probability, Neyman J. (ed.), 449-470.

Colleau J.J., L'Herminier P., Tanguy D., Felgines C., Le Mezec P., 1982a. Genetic parameters for type appraisal rating in French Friesian cattle breed. 2nd World Congress on Genetic applied to Livestock Production, Madrid, 4-8 October 1982, 7, 226-233, Editorial Garsi, Madrid. 
Colleau J.J., Dupont M., Muller A., Daburon E., Chambeyron J.J., Malterre C., 1982b. Young bull production as affected by divergent selection on milk yield. 2nd World Congress on Genetics applied to Livestock Production, Madrid, 4-8 October 1982, 7, 234-241, Editorial Garsi, Madrid.

Colleau J.J., Tanguy D., Boulanger P., Le Mezec P., 1982c. Prediction of diffusion of Holstein genes within the French Friesian population. 2nd World Congress on Genetics applied to Livestock Production, Madrid, 4-8 October 1982, 8, 69-74, Editorial Garsi, Madrid.

Cunningham E.P., Mc Clintock A.E., 1974. Selection in dual-purpose cattle populations : effect of beef crossing and cow replacement rate. Ann. Genet. Sel. Anim., 6, 227-239.

CunNingham E.P., Mololi B., 1982. Economic and genetic optimisation of dual purpose bull testing and selection. In: More O'Ferrall G.J. (ed.), Beef production from different dairy breeds and dairy beef crosses. (Curr. Top. Vet. Med. Anim. Sci., Martinus Nijhoff, The Hague, 21), 279-294.

DePotTer D., VANDEPrTte W., 1980. Programme de sélection pour des populations bovines à double fin : intensité de sélection optimale du point de vue génétique et économique sur la croissance après le test des performances. Rev. Agric., 33, 1257-1268.

DUTERTRE M., 1975. Modalités d'application, bilan et analyse du contrôle individuel des taureaux utilisés en insémination artificielle. Mémoire de fin d'études, Institut Supérieur Agricole de Beauvais, $50 \mathrm{pp}$.

DUTT J.E., 1973. A representation of multivariate normal probability integrals by integral transforms. Biometrika, 60, 637-645.

DUTT J.E., 1975. On computing the probability integral of a general multivariate t. Biometrika, 62, 201-205.

DUTT J.E., Soms A.P., 1976. An integral representation technique for calculating general multivariate probabilities with an application to multivariate $\chi^{2}$. Commun. Stat., 5, 377-388.

ELSEN J.M., Mocouot J.C., 1974. Recherches pour une rationalisation technique des schémas de sélection des bovins et ovins. Bull. Tech. Dep. Genet. Anim. I.N.R.A., 17, 97 pp.

ELSEN J.M., 1977. Pondérations économiques des indices de sélection dans le cas des populations de race mixte. Ann. Génét. Sél. Anim., 9, 73-85.

ELSEN J.M., 1980. Diffusion du progrès génétique dans les populations avec générations imbriquées : quelques propriétés d'un modèle de prévision. Ann. Génét. Sél. Anim., 12, 49-80.

GUPTA S.S., 1963. Bibliography on the multivariate normal integrals and related topics. Ann. Math. Stat., 34, 829-838.

GURLAND J., 1948. Inversion Formulae for the distribution of ratios. Ann. Math. Stat., 19, 228-237.

HILl W.G., 1971. Investment appraisal for national breeding programmes. Anim. Prod., 13, 37-50.

HrLL W.G., 1974. Prediction and evaluation of response to selection with overlapping generations. Anim. Prod., 18, 117-139.

Hinks C.J.M., 1970a. The selection of dairy bulls for artificial insemination. Anim. Prod., 12, 569-576.

HINKs C.J.M., 1970b. Performance test procedures for meat production amongst dairy bulls in AI. Anim. Prod., 12, 577-583.

JAIN J.P., AMBLE V.N., 1962. Improvement through selection at successive stages. J. Indian Soc. Agric. Stat., 14, 88-109.

LEBBE C., 1978. Les liaisons génétiques lait-viande intra-race chez les bovins laitiers - étude bibliographique. Mémoire de Diplôme d'Etudes Approfondies de Génétique Quantitative et Appliquée, Université Paris-Sud (Orsay), 82 pp.

LE MEZEC P., 1980. Utilisation des pointages de conformation laitière pour l'évaluation des taureaux Français Frisons (étude préliminaire). Mémoire de fin d'Etudes, E.N.S.F.A. Rennes, 91 pp.

LINDHE B., 1968. Model simulation of AI Breeding within a dual purpose breed of cattle. Acta Agric. Scand., 18, 33-41.

Maijala K., 1974. Many-sided progeny testing of bulls. Ann. Génét. Sél. Anim., 6, 253-266.

Miller R.H., LuKaszewicz M., Pearson R.E., 1981. Dual-purpose selection in dairy cattle. I. Genetic and phenotypic estimates. Z. Tierzüchtg. Züchtgsbiol., 98, 108-124. 
Milton R.C., 1972. Computer evaluation of the multivariate normal integral. Technometrics, 14, 881-889.

Mineur H., 1966. Techniques de calcul numérique. 606 pp., Dunod, Paris.

Mocouot J.C., Foulley J.L., 1973. Recherche des conditions de rentabilité d'un schéma de sélection d'une souche de bovins destinée au croisement de première génération pour la production de veaux de boucherie. Ann. Génét. Sél. Anim., 5, 189-209.

NiEBel E., 1974. Methodik der Zuchtplanung fur die Reinzucht beim Rind bei Optimierung nach Zuchtfortschritt und Zuchtungsgewinn. Dissertation Universität Hohenheim.

Petersen Ph., Gjol Christensen L., Bech Andersen B., Ovesen E., 1974. Economic optimisation of the breeding structure within a dual-purpose cattle population. Acta Agric. Scand., 24, 247-258.

Philipsson J., Janson L., Brannang E., 1975. Avelsindex for tjuver med avseende pa ekonomiskt betydelsfulla egenskaper. Lantbrkhoegs. Medd., 238, 1-31.

Rendel J.M., RobertSon A., 1950. Estimation of genetic gain in milk yield by selection in a close herd of dairy cattle. J. Genet., 50, 1-8.

SALZMANN F., 1978. Optimisation de la récolte et de la diffusion de taureaux d'insémination artificielle. Mémoire de fin d'études de l'Institut National Agronomique de Paris-Grignon.

Soller M., Bar Anan R., Pasternak H., 1966. Selection of dairy cattle for growth rate and milk production. Anim. Prod., 8, 109-119.

VINSON W.E., 1982. Selection for secondary traits in dairy cattle. 2nd World Congress on Genetics applied to Livestock Production, Madrid, 4-8 October 1982, 5, 370-386, Editorial Garsi, Madrid.

\begin{abstract}
Annexe
Calcul numérique de l'intégrale multiple d'une loi multinormale par la méthode de DutT (1973, 1975) et DutT \& SOMS (1976)
\end{abstract}

Soit à calculer :

$$
\begin{gathered}
\mathrm{L}_{\mathrm{k}, \mathrm{a}_{1} \ldots \mathrm{a}_{\mathbf{k}}}=\operatorname{Prob}\left(\mathrm{x}_{1}>\mathrm{a}_{1}, \ldots, \mathrm{x}_{\mathrm{k}}>\mathrm{a}_{\mathrm{k}}\right) \\
=\int_{\mathrm{a}_{1}}^{\infty} \ldots \int_{\mathrm{a}_{\mathbf{k}}}^{\infty} \mathrm{f}\left(\mathrm{x}_{1}, \ldots, \mathrm{x}_{\mathrm{k}}\right) \cdot \mathrm{dx_{1 }} \ldots \mathrm{d} \mathbf{x}_{\mathrm{k}} \\
=\int_{\mathrm{a}_{1}}^{\infty} \ldots \int_{\mathrm{a}_{\mathbf{k}}}^{\infty}(2 \pi)^{-\frac{\mathrm{k}}{2}} \cdot\left|\mathbf{R}_{\mathbf{k}}\right|^{-\frac{1}{2}} \cdot \exp \left[-\frac{1}{2} \mathbf{x}^{\prime} \cdot \mathbf{R}_{\mathbf{k}}^{-1} \cdot \mathbf{x}\right] \cdot \mathrm{d} \mathbf{x}
\end{gathered}
$$

où $\mathbf{f}\left(\mathrm{x}_{1}, \ldots, \mathrm{x}_{\mathrm{k}}\right)$ est la densité d'une loi $\mathrm{k}$-normale centrée réduite, $\left|\mathbf{R}_{\mathbf{k}}\right|$ est le déterminant de la matrice des corrélations $\mathbf{R}_{\mathbf{k}}$ entre les $\mathbf{k}$ variables normales $\mathbf{X}_{1}, \ldots, \mathbf{X}_{\mathbf{k}}$.

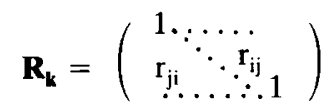


DuTT \& Soms (1976), utilisant une décomposition générale des intégrales multiples à bornes variables en somme d'intégrales sur ] $\mathrm{O},+\infty$ [, due à Gurland (1948), obtiennent dans le cas d'une loi multinormale :

$$
\begin{aligned}
& \mathrm{L}_{k, a_{1} \ldots \mathrm{a}_{\mathrm{k}}}=\left(\frac{1}{2}\right)^{\mathrm{k}}+\left(\frac{1}{2}\right)^{\mathrm{k}-1} \cdot \sum_{\mathrm{i}} \mathrm{I}_{1}\left(\mathrm{a}_{\mathrm{i}}\right)+\left(\frac{1}{2}\right)^{\mathrm{k}-2} \cdot \sum_{\mathrm{i}<\mathrm{j}} \sum_{2} \mathrm{I}_{2}\left(\mathrm{a}_{\mathrm{i}}, \mathrm{a}_{\mathrm{j}}\right) \\
& +\left(\frac{1}{2}\right)^{\mathrm{k}-3} \cdot \sum_{\mathrm{i}<\mathrm{j}<\ell} \sum_{3} \mathrm{I}_{3}\left(\mathrm{a}_{\mathrm{i}}, \mathrm{a}_{\mathrm{j}}, \mathrm{a}_{\ell}\right)+\ldots \\
& +I_{k}\left(a_{1}, \ldots, a_{k}\right) \\
& I_{m}=\frac{2}{(2 \pi)^{m}} \int_{0}^{\infty} \ldots \int_{0}^{\infty} \exp \left[-\frac{1}{2} \mathbf{t}^{\prime} \mathbf{t}\right] \cdot \frac{\mathrm{d}_{\mathrm{m}}(\mathbf{t})}{\prod_{\mathrm{r}=1}^{\mathrm{m}} \mathbf{t}_{\mathrm{r}}} \cdot \mathrm{dt} \\
& \left(t^{\prime}=\left(t_{1}, \ldots, t_{m}\right): \text { variables d'intégration }\right)
\end{aligned}
$$

avec

où $\mathrm{d}_{\mathrm{m}}$ est la somme des $2^{\mathrm{m}-1}$ premiers termes de la mième différence centrale de la partie réelle de $\left\{\mathbf{i}^{-m} \cdot \exp \left[-\mathbf{i t}^{\prime} \mathbf{a}-\frac{1}{2}\left(\mathbf{t}^{\prime} \mathbf{R t}-\mathbf{t}^{\prime} \mathbf{t}\right)\right]\right\}$ soit $d_{m}=\exp \left[-\left(r_{j_{1} j_{2}} \cdot t_{j_{1}} \cdot t_{j_{2}}+r_{j_{1} j_{3}} \cdot t_{j_{1}} \cdot t_{j_{3}}+\ldots+r_{j_{m-1} j_{m}} \cdot t_{j_{m-1}} \cdot t_{j_{m}}\right)\right]$

$$
\begin{aligned}
& \times \cos ^{*}\left(t_{j_{1}} \cdot a_{j_{1}}+t_{j_{2}} \cdot a_{j_{2}}+\ldots+t_{j_{m}} \cdot a_{j_{m}}\right) \\
& -\exp \left[-\left(r_{j_{1} j_{2}} \cdot\left(-t_{j_{1}}\right) \cdot t_{j_{2}}+r_{j_{1} j_{3}} \cdot\left(-t_{j_{1}}\right) \cdot t_{j_{3}}+\ldots+r_{j_{m-1} j_{m}} \cdot t_{j_{m-1}} \cdot t_{j_{m}}\right)\right] \\
& \times \cos ^{*}\left(\left(-t_{j_{1}}\right) \cdot a_{j_{1}}+t_{j_{2}} \cdot a_{j_{2}}+\ldots+t_{j_{m}} \cdot a_{j_{m}}\right)
\end{aligned}
$$

$(*)=\ll \cos »$ si m est pair

$=\ll \sin »$ si m est impair

Chacune des intégrales $I_{m}$ à calculer sur ] $O,+\infty$ [ est donc de la forme :

$$
A=\int_{0}^{\infty} \exp \left[-\frac{t_{1}^{2}}{2}\right] \cdot g\left(t_{1}\right) d t_{1}
$$

( $g\left(t_{1}\right)$ pouvant être elle-même une intégrale multiple). A est évaluée simplement par quadrature de Gauss (Booth, p. 41) :

$$
A=w_{1} \cdot g\left(h_{1}\right)+w_{2} \cdot g\left(h_{2}\right)+\ldots+w_{n} \cdot g\left(h_{n}\right)
$$

où les $w_{i}$ sont des facteurs de pondération et les $h_{i}$ les $n$ racines positives du polynôme d'Hermite de degré $2 n$. $h_{i}$ et $w_{i}$ ont été tabulés (MIneur, p. 322). La précision de l'évaluation de $\mathrm{A}$ dépend de $\mathrm{n}$ : pour $\mathrm{n}=4$, elle est généralement très bonne, à condition que les bornes $\left(\mathrm{a}_{\mathrm{i}}\right)$ ne soient pas trop extrêmes (inférieures à 2-2,5 en valeur absolue).

D'autre part, les intégrales des fonctions $d_{m}$ conduisent à une expression de $g\left(h_{i}\right)$ dans l'équation (1) qui est encore de la forme $g\left(h_{i}\right)=\int_{0}^{\infty} \exp \left[-\frac{t_{2}^{2}}{2}\right] \cdot g_{1}\left(t_{2}\right) \cdot d t_{2}$ que l'on peut de nouveau évaluer par quadrature de GAuss, et ainsi de suite. 
490

V. DUCROCQ

On aura par exemple :

$$
\begin{gathered}
I_{2}\left(a_{u}, a_{v}\right) \alpha \int_{0}^{\infty} \int_{0}^{\infty} \exp \left[-\frac{t_{1}^{2}+t_{2}^{2}}{2}\right] \cdot \frac{d_{2}\left(t_{1}, t_{2}\right)}{t_{1} \cdot t_{2}} \cdot d t_{1} \cdot d t_{2} \\
\alpha \int_{0}^{\infty} \exp \left[-\frac{t_{2}^{2}}{2}\right] \cdot\left(\sum_{i=1}^{n} \frac{w_{i}}{h_{i}} \cdot \frac{d_{2}\left(h_{i}, t_{2}\right)}{t_{2}}\right) \cdot d t_{2} \\
\alpha \sum_{i=1}^{n} \sum_{j=1}^{n}\left[\frac{w_{i}}{h_{i}} \cdot \frac{w_{j}}{h_{j}} \cdot d_{2}\left(h_{i}, h_{j}\right)\right]
\end{gathered}
$$

avec $d_{2}\left(h_{j}, h_{j}\right)=\exp \left[-r h_{j} h_{j}\right] \cdot \cos \left(h_{i} \cdot a_{j}+h_{j} \cdot a_{v}\right)$

$-\exp \left[r h_{j} h_{j}\right] \cdot \cos \left(-h_{i} \cdot a_{u}+h_{j} \cdot a_{v}\right)$ 